\title{
The effect of the temperature of graphene oxide reduction on low-temperature sorption of ${ }^{4} \mathrm{He}$
}

\author{
A.V. Dolbin, M.V. Khlistyuck, V.B. Esel'son, V.G. Gavrilko, N.A.Vinnikov, \\ and R.M. Basnukaeva \\ B. Verkin Institute for Low Temperature Physics and Engineering of the National Academy of Sciences of Ukraine \\ 47 Lenin Ave., Kharkov 61103, Ukraine \\ E-mail: dolbin@ilt.kharkov.ua
}

I. Maluenda, W.K. Maser, and A.M. Benito

Instituto de Carboquímica ICB-CSIC, 4 Miguel Luesma Castán, E-50018 Zaragoza, Spain

Received July 16, 2015, published online November 23, 2015

\begin{abstract}
The sorption of ${ }^{4} \mathrm{He}$ by graphene oxide powders thermally reduced at $T=200,300,500,700,900{ }^{\circ} \mathrm{C}$ has been investigated in the interval $1.5-290 \mathrm{~K}$. The measured dependence of the quantity of sorbed helium upon the reduction temperature shows up as a nonmonotonic curve. The highest quantities of helium were sorbed by the samples reduced at $T=300$ and $900{ }^{\circ} \mathrm{C}$. It is assumed that the thermal reduction of graphite oxide by heating it to $300{ }^{\circ} \mathrm{C}$ causes evaporation of the water intercalated in the spacings of the carbon layers, this results in exfoliation of the graphene planes, which enhances the sorptive capacity. Heating the samples to $900{ }^{\circ} \mathrm{C}$ generates numerous defects in the carbon planes, as a result, the interlayer spacings become accessible for sorption, which enhances the sorptive capacity.
\end{abstract}

PACS: 65.80.+n Thermal properties of small particles, nanocrystals, and nanotubes.

Keywords: graphene oxide, thermal reduction, ${ }^{4} \mathrm{He}$ sorption.

\section{Introduction}

The recent two decades have witnessed a significant advance in nanotechnologies which have become a new strategic trend in various fields of industry. Materials science owes its drastic changes to the advent of novel nanostructural forms of some substances, for example, allotropic carbon modifications that have been a particular concern of the scientific community. One of these is graphene, a new form of carbon which has been recently an object of the most extensive research.

Graphene is a two-dimensional single-layer carbon structure. Its surface consists of regular hexagons of side $1.42 \AA[1]$ with $s p^{2}$-hybridized carbon atoms at each vertex. This type of structure is a constituent of crystalline graphite in which graphene planes are about $3.35 \AA$ apart from one another [1].

The advent of a relatively simple technique of separating graphene as an individual sample and new macroscopically available graphene-based materials (graphene oxide, graphene oxide paper) have drawn special attention to pro- duction, investigation and application of graphene. This is due to the wide-range industrial potentialities of graphene allowed by its unique physical and chemical properties: high electric [2] and thermal conductivity [3], the dependence of the electronic characteristics on various-origin added radicals at the graphene surface [4], controllable energy gap width [5], quantum Hall effect [6], extraordinarily high-charge carrier mobility [7] and high elasticity.

These properties promise attractive applications of graphene as a basis for novel nanomaterials possessing improved mechanical, electric and thermal physical characteristics or as highly efficient gas [8,9] and biological $[10,11]$ sensors.

Graphene oxide consists of undamaged graphite domains with inclusions of $s p^{3}$-hybridized carbon atoms which contain functional hydroxyl and epoxy groups on the upper and lower surfaces of each graphene sheet. There are also inclusions of $s p^{2}$-hybridized carbon-containing carboxyl and carbonyl groups concentrated mainly at the edges of a graphene sheet. Graphene oxide, like graphite, has a layer structure (normally several carbon layers). The 
interlayer spacing varies within $6-8 \AA[12,13]$ depending on the employed preparation technique and the oxidation level of graphene oxide. The carbon layers in graphene oxide are deformed because of the $s p^{2} \rightarrow s p^{3}$ transitions of the carbon atoms. Graphene oxide has a rule abundant topological structure defects and ruptures. The layers of graphene oxide are bonded rather weakly.

At present thermal reduction is one of the most practiced techniques of preparing graphene-type materials on a commercial scale. Thermally reduced graphene oxide (TRGO) is prepared from graphene oxide (GO) [14,15]. In turn, GO is obtained from graphite using various chemical oxidizing agents $[13,16]$. Thermal reduction of graphene is an intriguing technology: on the one hand, it holds much promise of cost cuttings on mass TRGO production; on the other hand, a lack of chemical oxidizers is not improbable and can be a serious challenge. Besides, it is impossible to remove completely the traces of chemical oxidants from GO powders $[15,17,18]$, which may impede their special biological and medical applications. It is also important that residual oxide groups and surface defects inevitable on reducing graphene oxide affect significantly the structure of graphene planes. In this context reduced graphene oxide and graphene can hardly be considered as totally identical. Thermal reduction of graphene oxide is rather a complex process involving thermally activated multi-stage removal of the intercalated water molecules and oxide groups, such as carboxyl (-COOH), carboxyl and partially-hydroxyl (C-OH and $\mathrm{O}-\mathrm{H})$, epoxy $(\mathrm{C}-\mathrm{O}-\mathrm{C})$, surface single-bonded oxygen $(\mathrm{C}-\mathrm{O})$ and carbonyl $(\mathrm{C}=\mathrm{O})$ groups.

The presence of water and $\mathrm{O}_{2}$-containing groups has a significant effect on the physical properties of graphene oxide, including its sorptive characteristics. Owing to their large specific surface area $[19,20]$, graphene oxide and reduced graphene oxide are used as highly efficient sorbents. It is therefore topical to investigate the sorptive characteristics of GO and TRGO in a wide temperature range, particularly in regard of helium whose properties at low temperatures are largely due to quantum effects $[21,22]$.

\section{Experimental technique and results}

The sorptive characteristics of graphene oxide in respect of ${ }^{4} \mathrm{He}$ have been investigated as a function of the temperature of thermal graphene oxide reduction. The starting graphite oxide $(\mathrm{GtO})$ was obtained from graphite powder using the modified Hammers method. Its subsequent reduction and exfoliation were carried out through thermal heat- ing of $\mathrm{GO}$ in the atmosphere of argon. Five samples were treated and each of them was heated to a certain highest temperature $\left(200,300,500,700\right.$ and $\left.900{ }^{\circ} \mathrm{C}\right)$. The sorptive properties of the thermally reduced GO samples were investigated on a laboratory test bench (its design and operation are detailed elsewhere $[21,22])$. The temperature of the experiment was $1.5-290 \mathrm{~K}$. Prior to testing, the powder samples were evacuated for five days at room temperature in the measuring cell of the test bench, which was aimed at removing possible gas impurities and moisture.

Graphite oxide and the thermally reduced samples of graphene oxide were saturated with helium under the pressure $\sim 1$ Torr. The lowest temperature of the experiment was dictated by the ${ }^{4} \mathrm{He}$ gas pressure in the measuring system. In the course of saturation the pressure was maintained 2.5-3 times lower than the equilibrium pressure of saturated ${ }^{4} \mathrm{He}$ vapor at a particular temperature. As helium was sorbed, additional portions of ${ }^{4} \mathrm{He}$ were fed to the cell. This saturation procedure secured the system against ${ }^{4} \mathrm{He}$ vapor condensation and the formation of a film on the surfaces of the powder grains and the cell walls.

The supply of the ${ }^{4} \mathrm{He}$ gas was cut off when the equilibrium pressure $10^{-2}$ Torr was reached in the cell. The cell was then hermetically sealed and the changes in the pressure were registered during the ${ }^{4} \mathrm{He}$ desorption on stepwise heating of the sell. The ${ }^{4} \mathrm{He}$ gas released on heating was taken to an evacuated calibrated vessel whose internal pressure was measured using two capacitive MKS-627 pressure transducers which allowed pressure measurement in the range $10^{-3}-1000$ Torr, the error being $\pm 1 \cdot 10^{-4}$ Torr. Then the measurement procedure was repeated at the next temperature point. The temperature dependences of the ${ }^{4} \mathrm{He}$ quantities desorbed from GtO and TRGO samples are illustrated in Fig. 1.

The desorption was monitored over the interval $T=$ $=9-900 \mathrm{~K}$, however the sorbed helium was nearly fully desorbed from the samples on the heating to $24 \mathrm{~K}$. The dependences of the total quantity of desorbed ${ }^{4} \mathrm{He}$ upon the temperature of the sample reduction is shown in Fig. 2 and Table 1. In Figs. 1, 2 and in Table 1 the quantity of the desorbed ${ }^{4} \mathrm{He}$ atoms is normalized to the total quantity of $\mathrm{C}$ atoms in the samples.

Note that the total quantities of the sorbed and desorbed ${ }^{4} \mathrm{He}$ were equal within the experimental error. The dependence of the total quantity of sorbed ${ }^{4} \mathrm{He}$ upon the temperature of the sample reduction is described by a nonmonotonic curve. The largest quantities of the sorbed ${ }^{4} \mathrm{He}$

Table 1. The total ${ }^{4} \mathrm{He}$ concentrations in the GtO and TRGO samples at different reduction temperatures (quantity of desorbed He atoms $N_{\mathrm{He}}$ per total quantity of $\mathrm{C}$ atoms $N_{\mathrm{C}}$ in the samples)

\begin{tabular}{c|c|c|c|c|c|c}
\hline \hline Sample & GtO & TRGO-200 & TRGO-300 & TRGO-500 & TRGO-700 & TRGO-900 \\
\hline \hline$N_{\mathrm{He}} / N_{\mathrm{C}}$ & 0.00054 & 0.0063 & 0.022 & 0.018 & 0.0044 & 0.027 \\
\hline wt. $\%$ & 0.018 & 0.211 & 0.736 & 0.392 & 0.146 & 0.901 \\
\hline \hline
\end{tabular}



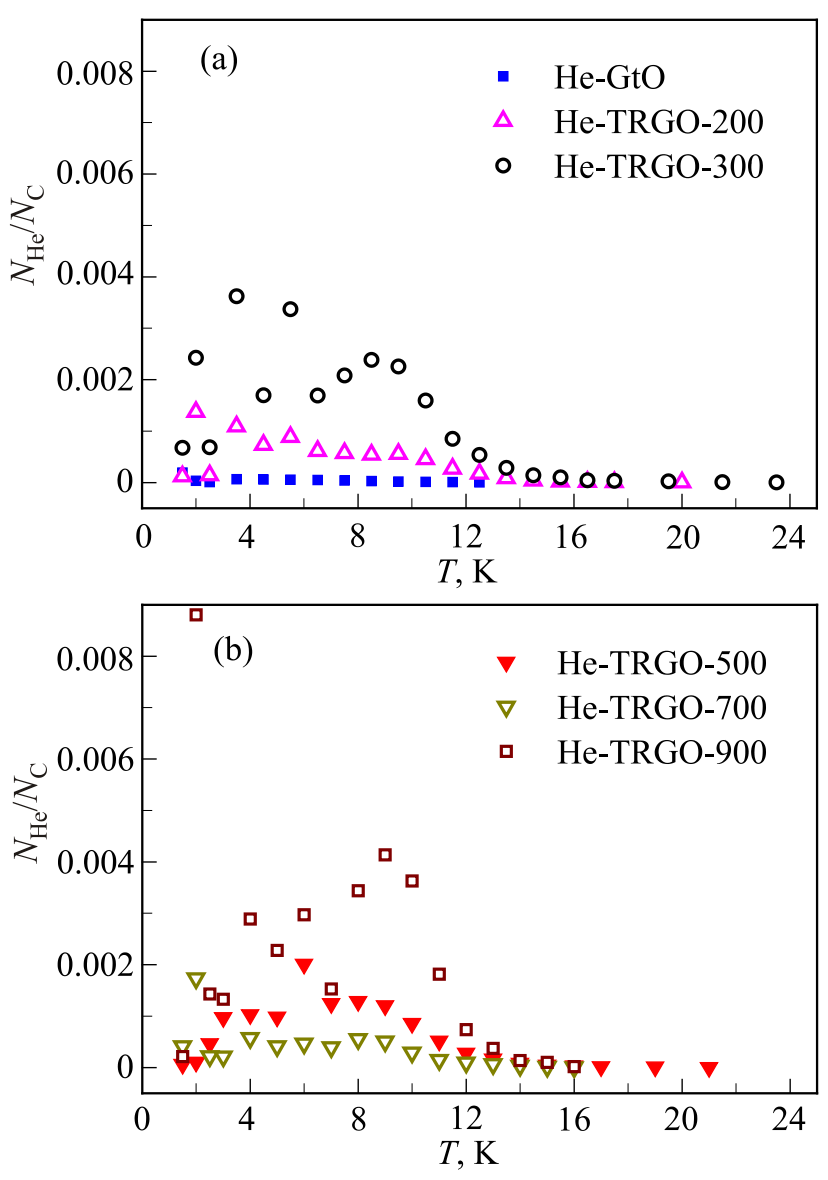

Fig. 1. (Color online) The temperature dependences of the relative ${ }^{4} \mathrm{He}$ quantities desorbed from the samples: (a) $\mathrm{GtO}(\mathbf{\square})$, TRGO reduced at $T=200{ }^{\circ} \mathrm{C}(\Delta), 300{ }^{\circ} \mathrm{C}(\mathrm{O})$; (b) TRGO reduced at $T=500{ }^{\circ} \mathrm{C}(\boldsymbol{\nabla}), 700{ }^{\circ} \mathrm{C}(\nabla)$ and $900{ }^{\circ} \mathrm{C}(\square)$.

were observed on the samples reduced at 300 and $900{ }^{\circ} \mathrm{C}$ (Fig. 2). The increased sorptive capacity of the sample heated to $300{ }^{\circ} \mathrm{C}$ may be attributed to the disordering of the layer structure in graphite oxide by evaporation of the intercalated water. On heating to $700{ }^{\circ} \mathrm{C}$ the $\mathrm{O}_{2}$-containing groups disappear, defects are produced and the spacings of the graphene layers decrease, which deteriorates the sorptive capacity of the samples.

The thermal treatment at a higher temperature $\left(900{ }^{\circ} \mathrm{C}\right)$ can destroy the residual $\mathrm{O}_{2}$-containing groups and produce a great number of defects [23]. As a result, the interlayer spacing in GO become accessible from sorption, which increases the sorptive capacity of the sample. The growing number of defects in the carbon planes at higher reduction temperatures show us another pronounced desorption maximum at $T \sim 10 \mathrm{~K}$ (Fig. 1(b), TRGO-500, TRGO-700 and TRGO-900 samples).

\section{Conclusions}

The desorption spectra characterizing the sorptive capacity of the samples of thermally reduced graphene oxide for helium have been taken in the interval $T=1.5-24 \mathrm{~K}$

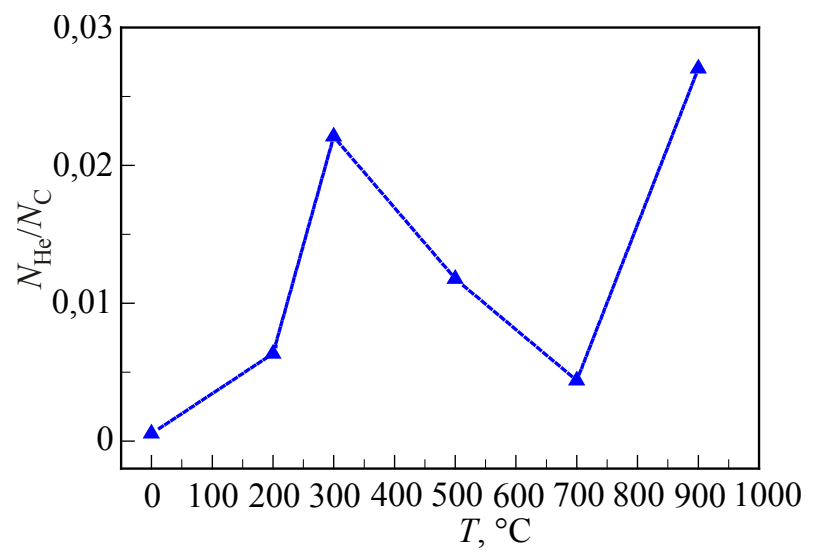

Fig. 2. The dependence of the relative quantity of sorbed helium on the temperature of the sample reduction.

using the method of thermoprogrammed desorption. The dependence of quantity of desorbed ${ }^{4} \mathrm{He}$ upon the temperature of the sample reduction has been plotted on the basis of the experimental results. It is assumed that the thermal reduction of graphite oxide by heating it to $300{ }^{\circ} \mathrm{C}$ in the Ar atmosphere causes evaporation of the water intercalated in the spacings of the carbon layers. This results in exfoliation of the graphene planes, which enhances the sorptive capacity. The thermal reduction at $700{ }^{\circ} \mathrm{C}$ suppresses sorptive capacity of graphene oxide because heating to $700{ }^{\circ} \mathrm{C}$ destroys $\mathrm{O}_{2}$-containing groups and reduces in the interlayer spacings in graphene. Heating the samples to $900{ }^{\circ} \mathrm{C}$ generates numerous defects in the carbon planes. As a result, the interlayer spacings become accessible for sorption, which enhances the sorptive capacity.

Financial support from Spanish Ministry MINECO and the European Regional Development Fund (project ENE2013-48816-C5-5-R), the Regional Government of Aragon and the European Social Fund DGA-ESF (project T66) and Targeted Comprehensive Fundamental Research Program of NASU (project 6/15-H) is gratefully acknowledged.

1. D.D.L. Chung, J. Mater. Sci. 37, 1475 (2002).

2. C. Lee, X. Wei, J.W. Kysar, and J. Hone, Science 321, 385 (2008).

3. A.A. Balandin, S. Ghosh, W. Bao, I. Calizo, D. Teweldebrhan, F. Miao, and C.N. Lau, Nano Lett. 8, 902 (2008).

4. X. Huang, X. Qi, F. Boey, and H. Zhang, Chem. Soc. Rev. 41, 666 (2012).

5. F. Guinea, A.H. Castro Neto, and N.M.R. Peres, Phys. Rev. $B$ 73, 245426 (2006).

6. K.S. Novoselov, Z. Jiang, Y. Zhang, S.V. Morozov, H.L. Stormer, U. Zeitler, J.C. Maan, G.S. Boebinger, P. Kim, and A.K. Geim, Science 315, 1379 (2007).

7. K.I. Bolotin, K.J. Sikes, Z. Jiang, M. Klima, G. Fudenberg, J. Hone, P. Kim, and H.L. Stormer, Solid State Commun. 146, 351 (2008). 
8. F. Schedin, A.K. Geim, S.V. Movozov, E.W. Hill, P. Blake, M.I. Katsnelson, and K.S. Novoselov, Nature Materials 6, 652 (2007).

9. B. Huang, Z.Y. Li, Z.R. Liu, G. Zhou, S.G. Hao, J. Wu, B.L. Gu, and W.H. Duan, J. Phys. Chem. C 112, 13442 (2008).

10. B.G. Choi, H. Park, M.H. Yang, Y.M. Jung, J.Y. Park, S.Y. Lee, W.H. Hong, and T.J. Park, Nanoscale 2, 2692 (2010).

11. K.J. Huang, D.J. Niu, J.Y. Sun, C.H. Han, Z.W. Wu, Y.L. Li, and X.Q. Xiong, Colloids and Surfaces B: Biointerfaces 82, 543 (2011).

12. A.B. Bourlinos, D. Gournis, D. Petridis, T. Szabo, A. Szeri, and I. Dekany, Langmuir 19, 6050 (2003).

13. M. Hirata, T. Gotou, S. Horiuchi, M. Fujiwara, and M. Ohba, Carbon 42, 2929 (2004).

14. W. Gao, L.B. Alemany, L. Ci, and P.M. Ajayan, Nature Chemistry 1, 403 (2009).

15. H.K. Jeong, Y.P. Lee, M.H. Jin, E.S. Kim, J.J. Bae, and Y.H. Lee, Chem. Phys. Lett. 470, 255 (2009).

16. W.S. Hummers and R.E. Offerman, J. Am. Chem. Soc. 80, 1339 (1958).
17. G.I. Titelman, V. Gelman, S. Bron, R.L. Khalfin, Y. Cohen, and H. Bianco-Peled, Carbon 43, 641 (2005).

18. H.K. Jeong, Y.P. Lee, R.J.W.E. Lahaye, M.H. Park, K.H. An, I.J. Kim, C.W. Yang, C.Y. Park, R.S. Ruoff, and Y.H. Lee, J. Am. Chem. Soc. 130, 1362 (2008).

19. G. Srinivas, J. Burres, and T. Yildirim, Energy Environ. Sci. 5, 6453 (2012).

20. G. Srinivas, J.W. Burress, J. Ford, and T. Yildirim, J. Mater. Chem. 21, 11323 (2011).

21. A.V. Dolbin, V.B. Esel'son, V.G. Gavrilko, V.G. Manzhelii, N.A. Vinnikov, and S.N. Popov, JETP Letters 93, 577 (2011).

22. A.V. Dolbin, V.B. Esel'son, V.G. Gavrilko, V.G. Manzhelii, N.A. Vinnikov, and S.N. Popov, Fiz. Nizk. Temp. 36, 1352 (2010) [Low Temp. Phys. 36, 1091 (2010)].

23. Seung Hun Huh, Thermal Reduction of Graphene Oxide, Physics and Applications of Graphene - Experiments, Dr. Sergey Mikhailov (ed.) (2011), ISBN: 978-953-307-217-3, InTech. 